\section{A newly developed lung nodule during chemotherapy for small cell lung cancer}

\section{CLINICAL PRESENTATION}

A 73-year-old man was admitted with dyspnoea and facial oedema. He was previously healthy with a smoking history of 40 pack-years. A CT scan of the chest showed a peribronchial soft tissue mass in the central portion of the right upper lobe and conglomerated enlarged multiple lymph nodes in the right hilar, subcarinal, paratracheal and prevascular areas with superior vena cava (SVC) obstruction (fig 1A). A focal fibrotic lesion was also noted on the right upper lobe above the lung mass, suggesting post-inflammatory change (fig 2A). Small cell lung cancer was confirmed by percutaneous needle aspiration biopsy. Two cycles of combination chemotherapy with etoposide $\left(100 \mathrm{mg} / \mathrm{m}^{2}\right.$, day $1-3$, every 3 weeks) and carboplatin (area under the curve 6 , day 1 , every 3 weeks) were given after 10 fractions of palliative radiotherapy to relieve SVC obstruction, leading to marked improvement (fig 1B). His dyspnoea and facial swelling also improved without any new symptoms such as fever or purulent sputum. However, a new $1.8 \mathrm{~cm}$-sized lung nodule with central low density appeared on the site where the focal fibrosis had previously existed (fig $2 \mathrm{~B}, \mathrm{C}$ ).

\section{QUESTION}

What is your diagnosis?

See page 697.

This case was submitted by:

\section{S Kang, ${ }^{1}$ G Y Noh, ${ }^{1}$ D H Choe, ${ }^{2}$ C H Kim, ${ }^{1}$ J C Lee ${ }^{1}$}

${ }^{1}$ Department of Internal Medicine, Korea Cancer Center Hospital, Seoul, Korea; ${ }^{2}$ Department of Radiology, Korea Cancer Center Hospital, Seoul, Korea

Correspondence to: Dr J C Lee, Department of Internal Medicine, Korea Cancer Center Hospital, 215-4 Gongneung-dong, Nowon-gu, Seoul 139-706, Korea; jclee@kcch.re.kr

\section{Competing interests: None}

Thorax 2009;64:682. doi:10.1136/thx.2008.106260
Figure 1 (A) Contrast-enhanced CT scan obtained at the level of the azygos arch showing a classical manifestation of small cell lung cancer with conglomerated lymphadenopathy in the right upper lobe peribronchial area and the mediastinum with narrowing of the superior vena cava. (B) CT scan at the same level obtained after chemotherapy showing that the tumour had almost completely resolved with restoration of the patency of the superior vena cava.

Figure 2 (A) CT scan obtained at the apical portion of the right upper lobe showing lung parenchymal scarring which remained stable before and after chemotherapy. (B) Follow-up CT scan after two cycles of chemotherapy showing development of a nodule at the site of the scarring. (C) CT scan of mediastinal window setting showing central low attenuation and rim enhancement of the nodule.
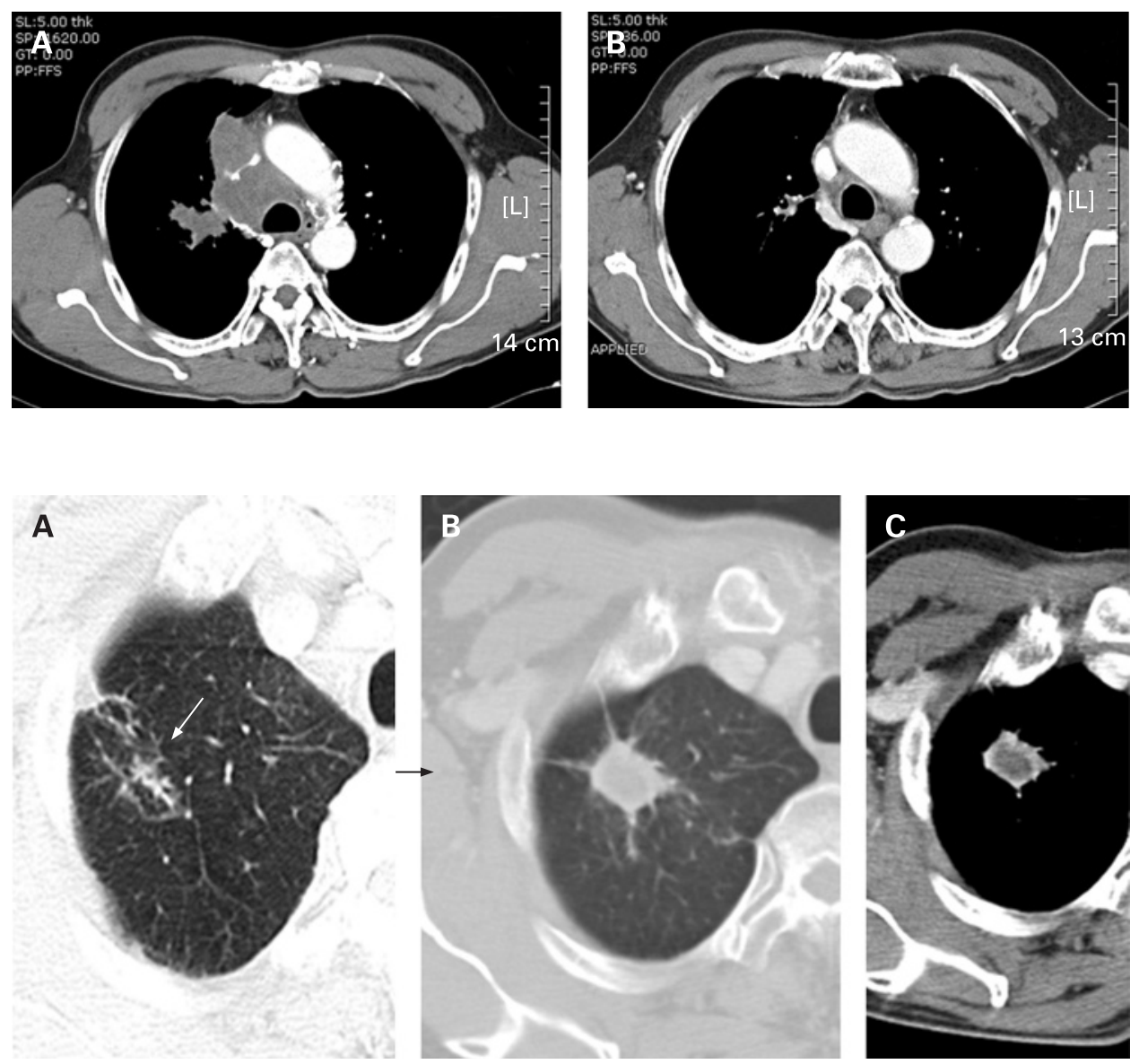